\title{
Effectiveness of dinoprostone vaginal pessary in induction of labour at term
}

\author{
Devdatt L. Pitale*
}

Department of Obstetrics and Gynecology, INHS Patanjali, Karwar, Karnataka, India

Received: 03 October 2017

Accepted: 10 October 2017

\section{*Correspondence:}

Dr. Devdatt L. Pitale,

E-mail: dipu.pitale@gmail.com

Copyright: () the author(s), publisher and licensee Medip Academy. This is an open-access article distributed under the terms of the Creative Commons Attribution Non-Commercial License, which permits unrestricted non-commercial use, distribution, and reproduction in any medium, provided the original work is properly cited.

\begin{abstract}
Background: Induction of labour is a very common obstetric procedure worldwide. The ultimate goal of induction of labour is to achieve a successful vaginal delivery. Dinoprostone is a Prostaglandin (PGE2) which acts on the collagen structural network of the cervix and makes it favourable, thus increasing the chances of a successful of a vaginal delivery. This study emphasizes on the importance of having a proper induction protocol in place and at the same time judicious use of the agents for induction of labour. This will help to reduce the maternal anxiety and stress associated with the induction of labour. The present study was undertaken to assess the effectiveness of dinoprostone vaginal pessary in induction of labour at term.

Methods: Twenty patients with unfavorable cervix at term were studied for the effectiveness of Dinoprostone vaginal pessary in induction of labor.

Results: Among the twenty patients 17 (85\%) delivered vaginally within 18 hrs and $3(15 \%)$ were in the active phase of labour. No untoward event was observed in any of the cases with a favorable neonatal outcome in all the cases.

Conclusions: Present study shows that Dinoprostone vaginal pessary is a highly effective method of induction of labor at term in properly selected cases. It reduces consistently the number of internal examinations and thus reducing the risk of ascending infections adding to it's safety along with reduced maternal anxiety associated with induction of labour.
\end{abstract}

Keywords: Dinoprostone, Induction of labour, Maternal anxiety, Vaginal pessary

\section{INTRODUCTION}

Induction of labour is a common procedure worldwide with overall rates in many countries now exceeding $20 \%$ of all births. ${ }^{1,2}$

Induction of labour is indicated when the risk of continuing pregnancy, for the mother or the fetus, exceeds the risk associated with induced labour and delivery. ${ }^{3}$

Preeclampsia $\geq 37$ weeks, significant but stable antepartum hemorrhage, chorioamnionitis, suspected fetal compromise and prelabour rupture of membranes at term are the high priority indications for induction of labour at term. The goal of induction of labor is to achieve a successful vaginal delivery. ${ }^{3}$

Many different methods have been used, but prostaglandins remain a preferred method for cervical ripening and labour induction. .,4,5 $^{1}$

Dinoprostone is a Prostaglandin (PGE 2) which acts on the collagen structural network of the cervix and makes it favourable thus increasing the chances of a successful of a vaginal delivery. 
Dinoprostone is the preferred form of prostaglandin and has been shown to increase the rate of vaginal delivery within $24 \mathrm{~h}$ and is generally given when the cervix has a Bishop's score of $\leq$ six. $^{6}$

Dinoprostone vaginal pessary is presented as a thin, flat semi-transparent polymeric vaginal delivery system which is rectangular in shape with rounded corners contained within a knitted polyester retrieval system containing $10 \mathrm{mg}$ of dinoprostone which is dispersed throughout it's matrix.

The advantages of the vaginal delivery system are reduction in the number of internal examination, improved patient compliance, easy to insert and easy to remove and an easy learning curve.

\section{METHODS}

This study included 20 pregnant women at term (37 completed weeks of gestation) attending the department of Obstetrics and Gynecology at INHS Asvini during November 2016 to February 2017.

Table 1: Indications for induction of labour.

\begin{tabular}{|lll|}
\hline $\begin{array}{l}\text { Indication } \\
\text { Prelabour rupture of } \\
\text { membranes }\end{array}$ & 10 & 50 \\
\hline Postdatism & 06 & 30 \\
\hline Preeclampsia & 03 & 15 \\
\hline Fetal distress & 01 & 05 \\
\hline
\end{tabular}

As shown in the Table 1, half of the cases $(50 \%)$ for induction of labour in the present study were prelabour rupture of membranes at term followed by postdatism. This are the high priority indications for the induction of labour worldwide. ${ }^{3}$

\section{Inclusion criteria}

- Singleton pregnancy

- Cephalic presentation

- Gestational age >37 weeks

\section{Exclusion criteria}

- $\quad$ Gestational age $<37$ weeks

- Previous uterine surgery

- Abnormal presentation

Twenty pregnant women who fulfilled the inclusion criteria were included in the study. A detailed case record was maintained of each and every case. A thorough clinical examination along with investigations like complete urine examination, hemogram and random blood sugar, obstetric usg and a 20 minutes non-stress test for fetal well-being were done. All the patients were informed in detail about the induction protocol and a written informed consent was taken.
Internal examination was done under strict aseptic precautions for assessment of cervix and Bishop's score was calculated. Dinoprostone vaginal pessary was inserted high into the posterior fornix under strict aseptic precautions in twenty pregnant women who satisfied the inclusion criteria, with a Bishop's score of $<6$. After the insertion of pessary they were asked to lie on bed for an hour. This allows the pessary to absorb vaginal moisture which makes it swell and prevents it from falling out. ${ }^{7}$ Patients were assessed after 18 hours from the time of insertion along with the monitoring of fetal heart unless indicated early due to uterine hyperstimulation, fetal heart rate abnormalities.

At least four regular uterine contractions during a 10minute observation period, along with the effaced cervix and os $>4 \mathrm{~cm}$ dilated on internal examination was considered as Active labor.

\section{RESULTS}

The Results were analyzed according to the maternal age and parity, induction-delivery interval and complications if any.

\section{Table 2: Parity of cases.}

\begin{tabular}{|lll|}
\hline Parity & No. of cases & Percentage \\
\hline Nulliparous & 15 & 75 \\
\hline Multiparous & 05 & 25 \\
\hline
\end{tabular}

As shown in the Table 2, majority of the patients were nulliparous $(75 \%)$. Rest five of the patients had at least one living issue delivered vaginally.

Table 3: Age distribution of the cases.

\begin{tabular}{|lll|}
\hline Age in years & Number of cases & Percentage \\
\hline $20-25$ & 05 & 25 \\
\hline $26-30$ & 12 & 60 \\
\hline$>30$ & 03 & 15 \\
\hline
\end{tabular}

Table 3 shows us the age distribution of the patients. $60 \%$ of the patients belonged to the age group of 26-30 years. Three patients in the present study belonged to the age group of $>30$ and were nulliparous.

Table 4: Gestational age distribution.

\begin{tabular}{|lll|}
\hline Gestational age in weeks & No. of cases & Percentage \\
$>37<40$ & 17 & 85 \\
\hline$>40$ & 03 & 15 \\
\hline
\end{tabular}

As shown in the above Table 4, 3 patients in the present study had gestational age of $>40$ weeks. $85 \%$ of the cases in this study belonged to the gestational age of $>37$ weeks and $<40$ weeks.

Majority of the patients (70\%) had a successful spontaneous vaginal delivery in the present study. 3 cases 
in this study delivered uneventfully by vacuum, due to failure of secondary powers. Remaining three patients were in active labour at end of 18 hours observation period.

Table 5: Mode of delivery.

\begin{tabular}{|lll|}
\hline $\begin{array}{l}\text { Mode of delivery } \\
\text { Spontaneous vaginal }\end{array}$ & 14 & 70 \\
\hline delivery & 03 & 15 \\
\hline $\begin{array}{l}\text { Assisted vaginal } \\
\text { delivery }\end{array}$ & 03 & Percentage \\
\hline
\end{tabular}

\section{Induction-delivery interval}

Average Induction-delivery interval in the present study was 18 hours. 3 patients (nulliparous with age $>30 \mathrm{yrs}$ ) who were in active labor after 18 hours, delivered vaginally after oxytocin acceleration. Absolutely zero cesarean section rate in this study adds to its merit and the importance of having a proper induction protocol in place.

\section{Neonatal outcome and complications}

All babies had an Apgar score of 10 at one minute. Uterine Hyperstimulation and diarrhoea was observed in one and two patients respectively. No foetal complications were observed in the present study.

Breastfeeding was established within half an hour of delivery in all the cases. Healthy mother and baby were discharged after neonatal immunization and screening along with necessary advice to the mothers about contraception and importance of exclusive breastfeeding.

\section{DISCUSSION}

Induction of labour is a very commonly performed intervention in Obstetrics. Proper selection of the cases for induction of labour and judicious use of the agents for induction of labour is thus very important.

Study conducted by Triglia MT et al, found spontaneous vaginal delivery in $72 \%$ patient with dinoprostone pessary as compared to gel preparation as compared to spontaneous vaginal delivery in $85 \%$ of cases in the present study. ${ }^{8}$ Assisted Vaginal delivery with vacuum was conducted in 3 patients for failure of secondary powers.

The average induction-delivery interval in this study was 18 hours. Rest of the three patients, nulliparous with age $>30$ years were in active phase of labour at the end of 18 hours and delivered vaginally with oxytocin acceleration. Recent studies have shown that Dinoprostone is the preferred form of prostaglandin and compared to placebo, vaginal PGE2 has been shown to increase the rate of vaginal delivery within 24 h. ${ }^{9,10}$ Metaanalysis conducted by M Ekaterina and et al showed that the risk of cesarean delivery following labour induction was significantly lower than the risk associated with expectant management. ${ }^{11}$

The present study shows that dinoprostone vaginal pessary is highly effective in induction of labor at term and even in postdated cases. Labour induction is associated with benefits for the fetus and no increased risk of maternal death. No untoward event was noted in any of cases throughout the course of labour before and after.

Uterine hyperstimulation and diarrhea was observed in one and two patients respectively. In these cases vaginal pessary was removed immediately under strict aspect precautions and the cervical findings in all the three cases were favourable.

The strength of the above study lies in the favourable neonatal outcome in all the twenty patients including one patient with foetal distress. These results are in line with the largest study to date by Kellie $\mathrm{T}$ et al that focusses primarily on the safety of dinoprostone vaginal pessary when used for more than 12 hours. This study has confirmed that use of dinoprostone vaginal pessary for up to $24 \mathrm{~h}$ is safe both in terms of uterine tachysystole and neonatal and maternal morbidity. ${ }^{12}$ Past studies have suggested that it can be left in longer with no apparent detrimental effects. ${ }^{13,14}$

Failed induction of labour results in cesarean section which adds to the stress of both mother and the obstetrician. ${ }^{15}$ This study had a successful induction of labour in all the cases thus reducing the maternal stress and anxiety associated with the induction of labour.

\section{CONCLUSION}

Dinoprostone vaginal pessary is highly effective in the induction of labour at term in properly selected cases. It reduces consistently the number of vaginal examinations and thus reducing the risk of ascending infections adding to its safety along with reduced maternal anxiety associated with induction of labour. Further research is needed to determine the cost effectiveness.

\section{ACKNOWLEDGMENTS}

This research was supported by the Indian Naval hospital ship, Asvini. Author would like to thank colleagues from Asvini for their constant support. Author also would like to thank Surgeon Commodore Dr Anupam Kapur, Head of the department of Obstetrics and Gynecology, Asvini for his assistance with methodology and for comments that greatly improved the manuscript.

\section{Funding: No funding sources}

Conflict of interest: None declared

Ethical approval: The study was approved by the Institutional Ethics Committee 


\section{REFERENCES}

1. American College of Obstetricians and Gynecologists. ACOG practice bulletin. Clinical Management Guidelines for ObstetricianGynecologists. No. 107. Induction Labor Obstet Gynecol. 2009;114:386-97.

2. Chauhan SP, Ananth CV. Induction of labor in the United States:a critical appraisal of appropriateness and reducibility. Semin Perinatol. 2012;36:336-43.

3. SOGC Clinical Practical Guideline. Induction of labour. J Obstet Gynecol Can. 2013;35(9).

4. National Institute for Health and Care Excellence. Induction of labour. (Clinical guideline 70). NICE, 2008. Available at www.nice.org.uk/guidance/cg70.

5. Tang J, Kapp N, Dragoman M, de Souza JP. WHO recommendations for misoprostol use for obstetric and gynecologic indications. Int $\mathbf{J}$ Gynecol Obstet. 2013;121:186-9.

6. Tenore JL. Methods for cervical ripening and induction of labor. Am Fam Phys. 2003;67(10):2123-8.

7. Jessop W. Induction of labor. Sheffield teaching Hospitals NHS Foundation trusts: November 2011. Available at http://www.sth.nhs.uk/clientfiles/File/pd5110_Induct ionOfLabour[1].pdf

8. Triglia MT, Palamara F, Lojacono A, Prefumo F, Frusca T. A randomized controlled trial of 24- hour vaginal dinoprostone pessary compared to gel for induction of labor in term pregnancies with a Bishop score $\leq 4$. Acta Obstetricia et Gynecologica Scandinavica. 2010 May 1;89(5):651-7.

9. Wing D. Techniques of cervical ripening prior to labor induction. UpToDate Online 19.1, viewed 7
April 2011. Available at http://www.uptodate.com/contents/techniques-forcervical- ripening-prior-to-labor-induction.

10. Kelly AJ, Malik S, Smith L, Kavanagh J, Thomas J. Vaginal prostaglandin (PGE2 and PGF2a) for induction of labour at term. Cochrane Database Syst Rev. 2009 Oct 7;(4):CD003101.

11. Mishanina E, Rogozinska E, Thatthi T, Uddin-Khan $\mathrm{R}$, Khan KS, Meads C. Use of labour induction and risk of cesarean delivery: a systematic review and meta-analysis. Canadian Med Assoc J. 2014 Jun 10;186(9):665-73.

12. Tathem K, Harris LJ, O'rourke P, Kimble RM. Dinoprostone vaginal pessary for induction of labour: Safety of use for up to $24 \mathrm{~h}$. Aust N Z J Obstet Gynecol. 2012 Dec 1;52(6):582-7.

13. Kho E, Sadler L, McCowan L. Induction of labour: A comparison between controlled-release dinoprostone vaginal pessary (Cervidil(r)) and dinoprostone intravaginal gel (Prostin E2(r)).Aust N Z J Obstet Gynaecol. 2008;48:473-7.

14. Wing DA. For the misoprostol vaginal insert consortium. misoprostol vaginal insert compared with dinoprostone vaginal insert. Obstet Gynecol. 2008;112:801-12.

15. Australian Council of Healthcare Standards. 2011. Available at http://www.achs.org.au/aboutus.

Cite this article as: Pitale DL. Effectiveness of dinoprostone vaginal pessary in induction of labour at term. Int J Reprod Contracept Obstet Gynecol 2017;6:5528-31. 